\title{
In silico Modeling Personalized Therapy of Pulmonary Artery Hypertension
}

\author{
Xiaonan Ying ${ }^{1}$, Wenqin $\mathrm{Li}^{2}$, Yan Wang ${ }^{3}$, Biaoru $\mathrm{Li}^{4 *}$ \\ ${ }^{1}$ Student, Department of Bioinformatics, Northeastern University, Boston, MA 02115, USA \\ ${ }^{2}$ Student, Department of Chemistry, University of California, Irvine, CA, USA \\ ${ }^{3} \mathrm{MD}$, Department of Internal Medicine, China Petroleum Kuerla Hospital, Kuerla, XinJiang, P.R. China \\ ${ }^{4}$ Senior Scientist, Department of Pediatrics and GA Cancer Center, Children Hospital at GA, Augusta, GA, USA
}

*Address for Correspondence: Dr. Biaoru Li, Senior Scientist, (Tenured), CN4111 Building, Department of Pediatrics and GA Cancer Center, Children Hospital at GA Medical College at Augusta, GA, USA

E-mail: bli@augusta.edu

Received: 19 Dec 2018/ Revised: 15 Mar 2019/ Accepted: 29 Jun 2019

\begin{abstract}
Background: The pulmonary arteries are the blood vessels that carry blood from the right side of the heart through the lungs. Pulmonary arterial hypertension (PAH) is a progressive disorder characterized by higher pressure of lungs pulmonary artery for no apparent reason. PAH is treatable although there is no clear cure for the disease. Fortunately, human genomics have been decoded in 2004 and several molecular targeting compounds related to PAH were discovered, the uncured disease should give clinical scientists and medical doctors new scenery to develop some new modules to administer patients.

Methods: We used a set of genomic data from clinical PAH to combine traditional medication and molecular target therapy so that integration modules will be used to the clinical field. The integration model had primarily relied on system biology including network, topology and gene-drug interaction database.

Results: In this research article, we firstly analyzed using genomic expression signature from a set of clinical PAH genomic data and three known PAH pathways and then we combined current medications and molecular targeting therapy into the integration model.

Conclusion: In the near future, we will develop a second-generation model based on the module by using individual clinical genomic data from different patients such as patient genomic data, clinical information including patient symptom and laboratory results.
\end{abstract}

Key-words: Gene expression signature, Integration medicine, Pulmonary arterial hypertension, Personalized therapy, Topology

\section{INTRODUCTION}

German doctor E. Romberg first reported PAH in 1891, who described a patient with thickening pulmonary artery but no heart or lung disease under autopsy ${ }^{[1]}$. Now we have known that PAH is a progressive disorder characterized by hypertension in the lungs for no apparent reason. Symptoms of PAH include dyspnea during exercise, chest pain and fainting episodes. PAH patients may experience several years without the

\section{How to cite this article}

Ying X, Li W, Wang Y, Li B. In silico Modeling Personalized Therapy of Pulmonary Artery Hypertension. SSR Inst. Int. J. Life Sci., 2019; 5(4): 2341-2348.

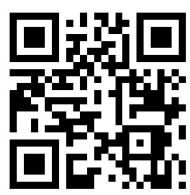

diagnosis ${ }^{[2]}$. However, it is important to cure PAH because of without optimal treatment, high blood pressure in the lungs causes the right heart to work much harder, progressively and eventually, the patient heart muscle may result in failure ${ }^{[3]}$.

Since 2004, when human genomics are decoded, it gives an expectation for medical doctors to treat the uncured disease. Furthermore, because several molecular targeting compounds related to PAH were discovered, it will be possible that PAH should be cured ${ }^{[4]}$. Foremost, system biology and gene-drug interaction databases are emerging; an integration model based on genomics will be possible for the combination employment of different medication methods in the clinical disease. Here we first studied a set of genomic databases from PAH for the integration model. Secondly, some comprehensive 
pathways are studied to $\mathrm{PAH}$ network mechanism so that we further set up a network construction by genomic expression signature obtained from the public GEO database. Finally, we combine PAH networks with current medication and PAH related to molecular targeting treatment, and therefore this integration modeling will be optimal to treat PAH.

The manual purpose will provide the possibility for effective treatment to this kind of uncured disease. In near future, we will continue to develop secondgeneration module with clinical genomic data from an individual patient and his/her information relied on each patient symptom and laboratory results. Our final purpose is that the feasible module can be used for MD to treat or medical care to maintain a reasonable quality of life according to personal genomics information, patient symptom and lab results.

\section{MATERIALS AND METHODS}

Clinical genomic sources- There were several public PAH genomic databases published in Gene Expression Omnibus (GEO). After these databases in GEO were carefully studied, we mainly selected GSE113439 from GEO for our study in silico model because the data based on diagnosis were defined by pre-capillary pulmonary hypertension. GSE113439 were studied from fifteen patients with PAH and eleven normal controls. The PAH group included six patients with idiopathic PAH, four patients with PAH secondary to connective tissue disease (CTD), four patients with PAH secondary to congenital heart disease (CHD) and one patient with chronic thromboembolic pulmonary hypertension (CTEPH) ${ }^{[5]}$. After collecting the genomic data, NIH BRB Array Tool (https://brb.nci.nih.gov/BRB-ArrayTools/ download.html) was plug into Excel for genomic expression profile mining.

\section{Topology analysis for personalized therapy modeling- As} our previous reports, we had got the genomic expression profiles as well as firstly studied this genomic expression profiles under the topology model. In details, the specific PAH gene expression profile after BRB tool mining was input into Cytoscape to observe abnormal expression (gene expression signature, GES) for the genomic characteristics. Based on our previous publications, we selected three indexes, betweenness Centrality (BC), which was a short pathway between two proteins (node), Connectivity Degree $(C D)$, which is a protein linking other protein number and Cluster Coefficient (CC), which means side-way to a protein. Furthermore, the topology formula selected in the network combined to the vessel endothelium pathway, all GES and vessel pathway laid the foundation for the establishment of operational therapeutic targets.

After we were studied the GES topology and also collected three pathways related with PAH: Nitric oxide pathway (NO), which helped to control blood pressure by opening arteries (also known as dilation) when needed; Endothelin pathway, which was almost the opposite of nitric oxide. It increases blood pressure and makes the blood vessels firm; Prostacyclin pathway, which helps with dilation and it helps prevent the vessels from getting blocked ${ }^{[6-10]}$.

GES and three pathways related to PAH be also input into a drug-bank in the Drug Genomic Interaction Database (DGIdb) to define targeted therapeutic drug and the targeting molecule. We were also studied an index from each compound with higher $\mathrm{BC}$ and lower $\mathrm{CC}$ and $\mathrm{CD}$. These targets indicate as a higher targeting for abnormal cells with lower toxicity for normal cells. Eventually, a list of compounds from drug-bank established to link genes, especially include FDA-approved drug and molecular therapeutic antibodies and small molecule therapeutics. This led to the establishment of compartment and drug response networks based on the abnormal genome expression characteristics obtained from PAH for the further purpose of personalized medication.

Topology analysis for targeting treatment- Three pathways have been discovered for PAH networks as introduced above, NO pathway, endothelin pathway, and prostacyclin pathway. When patients become PAH, the three pathways with their regulation will become major factors to involve in the disease ${ }^{[11-13]}$. We were applied for the list of molecular targets approved by FDA with targeting genes to combine into the network including three pathways and list of molecular targets.

Topology analysis for current medicine- Moreover, routine clinical health care for $\mathrm{PAH}$ focuses on an oxygen intake to increase NOS, decrease sodium to decrease vessel constriction, digoxin to increase vessel dilation and diltiazem and dihydropyridine to regulate calcium function ${ }^{[13]}$. Therefore, the topology analysis from current medication includes gene expression and their treatment. 
Topology analysis for integration module- As eventual combination for integration model, we merged all networks from genomic data including their therapeutic targets; three pathways with suppressing and activation related medication.

Support analysis- In order to support the module of the selected pathways for current drugs and targeted molecule therapy for personalized therapy, python scripts with their compartment to stimulate to assay a drug. The python scripts were established as our previously reported, they were used to stimulate $\mathrm{PAH}$ drugs to support the module and analyzed the matched therapeutic targets including traditional medicine and targeting treatment in the $\mathrm{PAH}$ network for targeted gene expression and the discovered therapeutic molecules. The design principle was that network with a dynamic model based on differential equations including qualitative relationships and directed responses as our previous report.

\section{RESULTS}

Construction and topology establishment from GESRecently, therapeutic targeting was going to focus on topology based on genomic expression profile to discover drug targeting, small molecule targeting, Ab targeting and RNA-interfering therapy. Base on the conception from our long-term data analysis and experimental support, although 15 specimens from patients with $\mathrm{PAH}$ to 11 control specimens were obtained from GEO data, we needed further refine a gene profile for construction for feasible therapeutic targets. After gene expression mining and analysis, 427 genes were refined for further study as Fig. 1.

15 PAH specimens vs 11 control specimens

2508 genes mined after 2 fold change

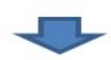

630 genes were mined after $>2$ fold change

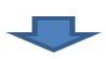

503 genes were defined with gene code

427 genes were refined after analysis

Fig. 1: A Gene expression mining and protocol was performed by BRB platform
Our laboratory has spent more than twenty years to study different topology parameters relied on our experimental assays such as quantitative rtPCR and Western blot. Although most of the parameters can be used in different cell-lines, animal and human beings in different labs, as our previous studies, both BC and DC majorly play an important role from clinical specimens while DC is likely to be toxic for normal cells such as normal lymphocytes due to their system-wide influence, thus the high $B C$ value indicates a significant targeting node from abnormal cells and low DC and CC means very few branches without their system-wide influence to cause normal cell dysfunction. After gene expression profile setup, we were firstly studied the GES, GES results. Due to study $\mathrm{PAH}$, we additionally input angiogenesis pathway and three $\mathrm{PAH}$ pathways into Cytoscape, a construction from both PAH GES and angiogenesis pathway/three PAH pathways were established as Fig. 2. The uncovered nodes (or genes or proteins) were loaded into the DGIdb to mine drugs, small molecule and other molecular therapy agents. The resulting node and drug candidates with their index (BC, DC, and $C C)$ were configured by the construction map as Fig. 3 because FDA has approved for several drugs of targeting therapy, we have not further studied new targeting, in details, in the DGIdb for clinical module purpose.

Topology and results of analysis for target medicine treatment- According to current efforts, the PAH targeting therapy, Riociguat, Sildenafil, and Tadalafil can be increased to the NO pathway, Epoprostenol, Treprostinil, whereas lloprost can be achieved mimics or increase PGI2 in prostacyclin pathway. Bosentan and Macitentan can blocked to ETB and ETA in endothelin pathway. In order to study $\mathrm{PAH}$ related to specific targeting therapy, we input three $\mathrm{PAH}$ pathways related to their specific therapy into Cytoscape, a resulting construction from both $\mathrm{PAH}$ pathways and targeting therapy was established as Fig. 4. 


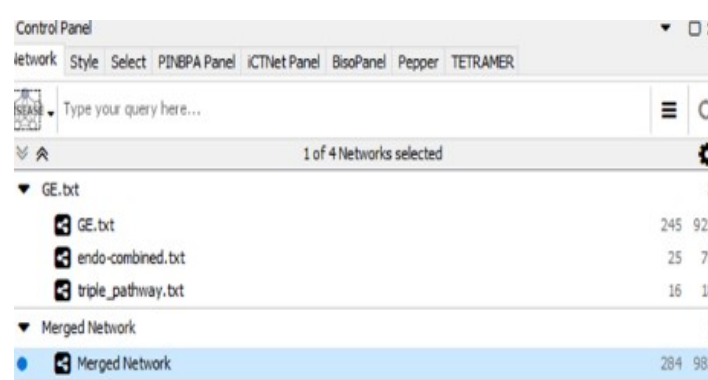

Fig. 2: Construction defined by Cytoscape platform depending on GES, endothelium pathway, three pathways (NO pathway, Prostacyclin pathway and Endothelin pathway) related with PAH

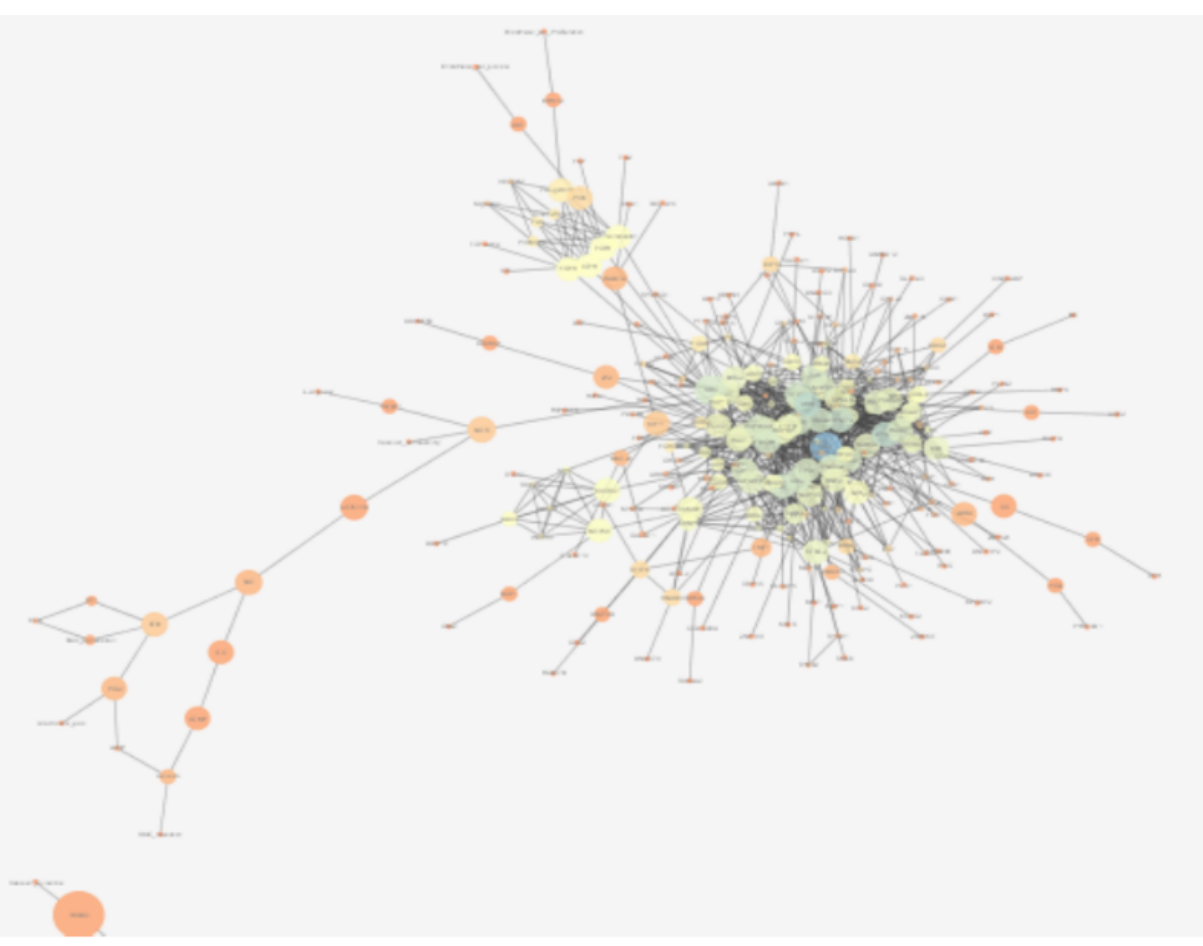

Fig. 3: Diagram defined by Cytoscape platform depending on GES, endothelium pathway, three pathways related with $\mathrm{PAH}$. The configuration was used for the topology analysis such as BC, DC and CC. For example, large node size means larger $\mathrm{BC}$ value and $\mathrm{DC}$ large means color dark 


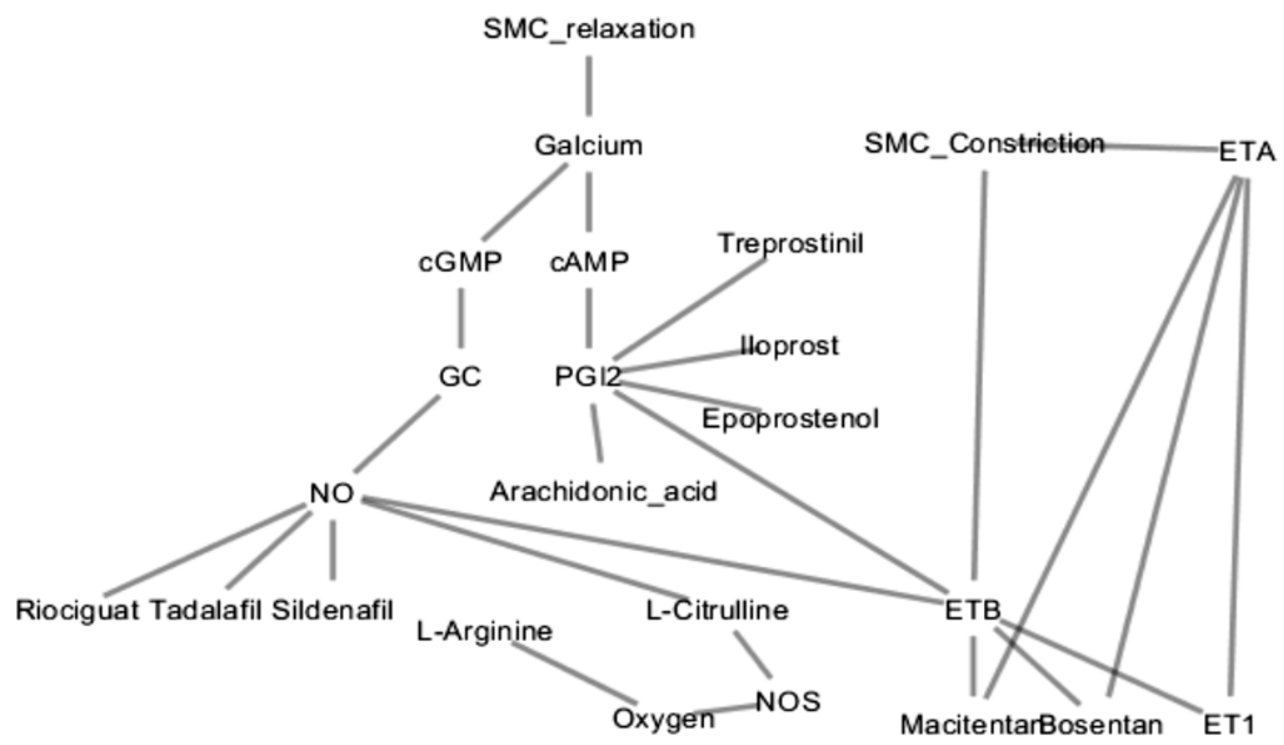

Fig. 4: Diagram was defined by Cytoscape platform depending on three pathways related with PAH with their targeting medication

Topology analysis and results for current medicineRoutine health care in hospital focuses on an oxygen intake, decrease sodium, digoxin and block-calcium channel (BCC) treatment. In order to combine the routine health care for topology system, we also combined three PAH pathways, vessel endothelium pathway and the common treatment such as an oxygen intake to increase NOS, decrease sodium to decrease vessel constriction, digoxin to increase vessel dilation and diltiazem and dihydropyridine to block calcium function. After the two pathways and common drugs input into Cytoscape, a resulting construction from both pathways and common health care was established as Fig. 5.

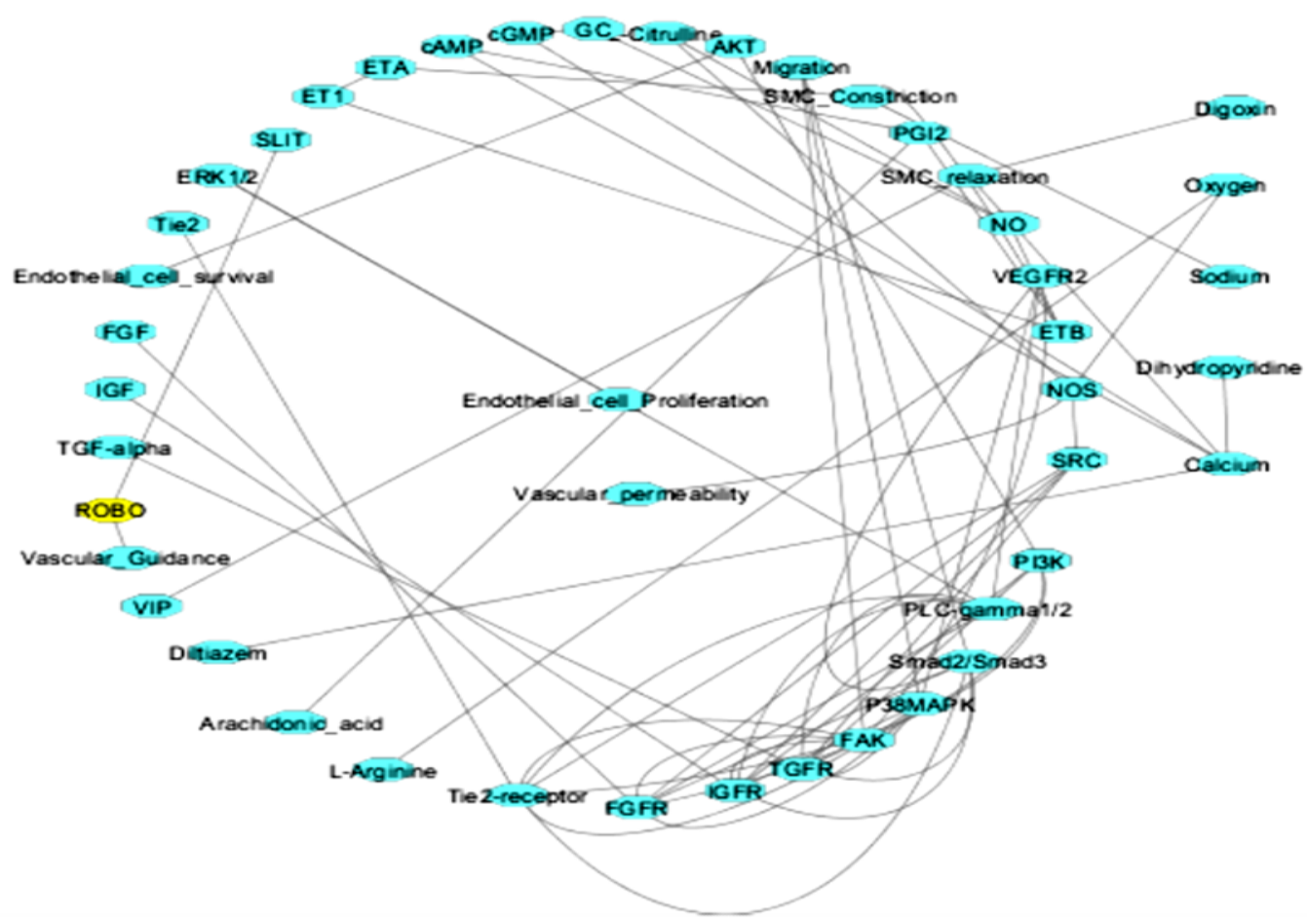

Fig. 5: Diagram indicated that an integration process including endothelium pathway, three pathways related with PAH with their current medication 


\section{Construction and topology analysis for integration} model- After we achieved construct from genomic data and endothelium pathway with their therapeutic targets, second construct from current PAH mechanism with specific targets, third construct from PAH mechanism with their common treatment, as Fig. 6, we merged all nodes within configuration, so that resulting node and drug candidates with their index $(\mathrm{BC}, \mathrm{DC}$, and $\mathrm{CC})$ were discovered by the construction map. As the Fig. 6 show, if we have a set of GES data, we can predict a comprehensive treatment, including current feasible administration and targeting treatment, which can administer PAH with their different symptoms.
Python analysis- In order to support the integration model for these selected pathways and their targeted drugs and a targeted molecule therapy including their current medication and their targeted drugs, a python scripts which was established in our lab are used to simulate the anti-PAH drugs in the module analyzing their therapeutic targets within common and targeting medication in the construct network. As Fig. 7, for example, if genomic data as GES was defined as harvested in the manual, three treatment measurement including oxygen intake, digoxin, and silde nafilcan be used to the personalized therapy.

Fig. 6: Diagram indicated that an integration model including genomics data with their GES, endothelium pathway, three pathways related with PAH with their medication and targeting therapy
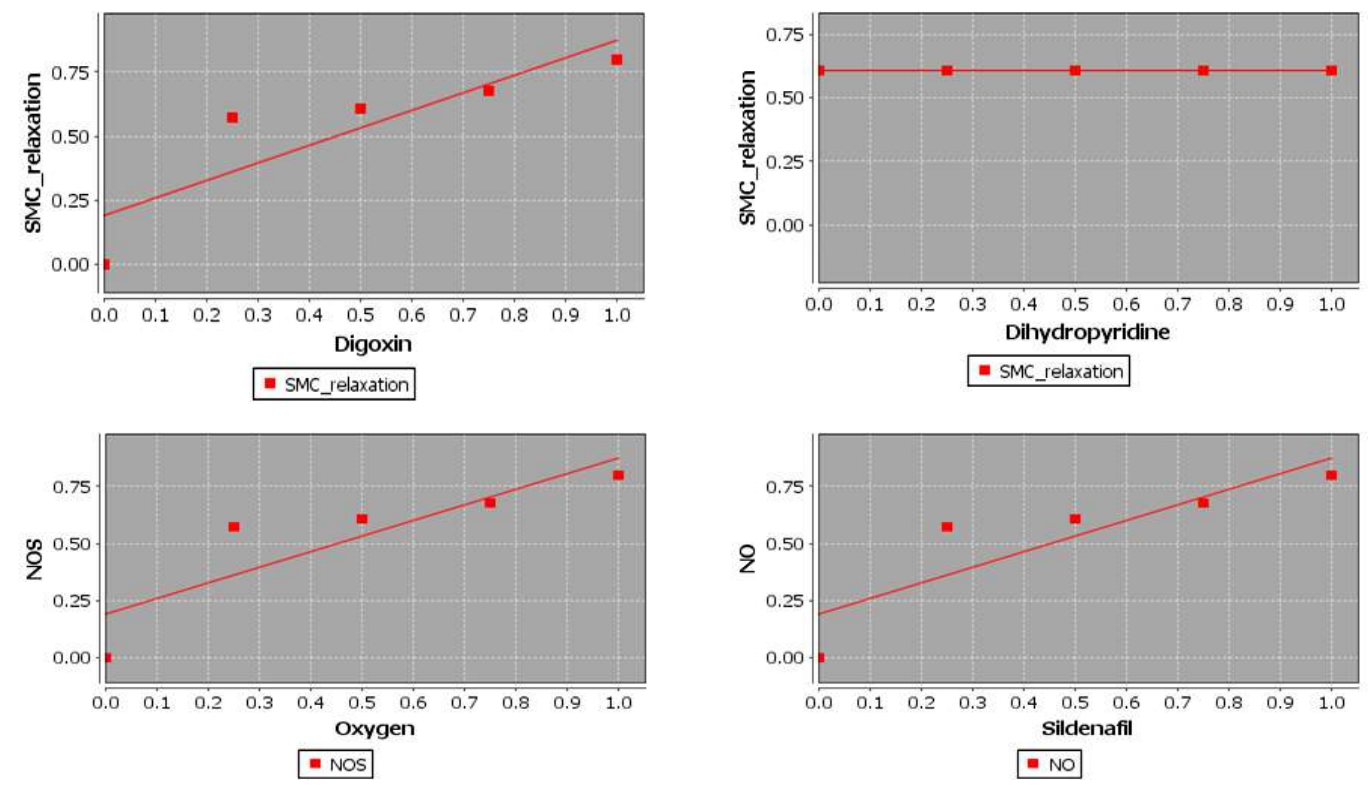

Fig. 7: Python analyses support that an integration results such as Oxygen intake increase NO, Digoxin can decrease pulmonary pressure and Sildenafil can increase NO system 


\section{DISCUSSION}

Pulmonary arterial hypertension (PAH) is a progressive disorder characterized by hypertension in the lungs. However, PAH has not any individual treatment for the high blood pressure in the lungs. The manual will provide the possibility for effective treatment to this kind of un-cured disease relied on genomic data. After that we studied a set of PAH genomic data, we set up a construction combined current medication and targeting treatment to configure this integration modeling in silico. Pulmonary arterial hypertension (PAH) is a progressive disorder characterized by hypertension in the lungs ${ }^{[14]}$. Since the discovery of endothelin-1 for last thirty years, the therapeutic strategy has applied in treatment of pulmonary arterial hypertension ${ }^{[15]}$. Up to now, more and more targeting receptors are going on marketing, for example, small molecules, monoclonal antibody antagonists and selective peptide agonists and antagonists ${ }^{[16]}$. This manual has provided a rationale for personal information such as personal genomics to stratify patients for allocation to treatment and highlights the potential to use personalized precision medicine in the PAH field. The manual will provide the possibility for effective treatment to this kind of uncured disease relied on genomic data. After we studied a set of $\mathrm{PAH}$ genomic data, we set up a construction combined current medication and targeting treatment to configure this integration modeling in silico.

\section{CONCLUSIONS}

Since the integration model in silico was developed by a set of genomic data from $\mathrm{PAH}$ as the manual, in near future, we will further develop second-generation module with clinical genomic data from an individual patient with that clinical information including each patient symptom, laboratory results and so on. Our final purpose is that the feasible module will be set up to be used for clinical MD to treat or medical care to maintain a reasonable quality of life for each patient suffering from $\mathrm{PAH}$.

\section{ACKNOWLEDGMENTS}

Under the support of Dr. H. D. Preisler, we have set up different methods and models to analyze genomic profiles such as CD3, CD4 and CD8 from immune and tumor diseases related to personalized therapy. This clinical application was previously supported by National Cancer Institute IRG-91-022-09, USA (to BL).

\section{CONTRIBUTION OF AUTHORS}

Research Concept- Biaoru Li, Yan Wang

Research Design- Biaoru Li, Yan Wang

Supervision- Yan Wang

Materials- Yan Wang

Data collection- Wenqin Li

Data analysis and Interpretation- Wenqin Li

Literature search- Xiaonan Ying

Writing article- Xiaonan Ying, Biaoru Li

Critical review- Yan Wang

Article editing- Xiaonan Ying

Final approval- Biaoru Li

\section{REFERENCES}

[1] Malakan RE, Pouraliakbar HR. Isolation of the left brachiocephalic artery revisited: A 52-year literature review and introduction of a novel anatomic-clinicalprognostic classification. Ann. Pediatr. Cardiol., 2019; 12(2): 117-29.

[2] Sakuma M, Toyoda S, Inoue T, Node K. Inflammation in pulmonary artery hypertension. Vascul. Pharmacol., 2019; 106562.

[3] Farber HW, Loscalzo J. Pulmonary arterial hypertension N. Engl. J. Med, 2004; 351: 1655-65.

[4] Humbert M, Ghofrani HA. The molecular targets of approved treatments for pulmonary arterial hypertension. Thorax, 2016; 71: 73-83.

[5] Mura M. Gene expression profiling of pulmonary arterial hypertension. Gene Expression Omnibus, 2018; 4-12.

[6] Humbert M, Sitbon O, Simonneau G. Treatment of pulmonary arterial hypertension. N. Engl. J. Med., 2004; 351: 1425-36.

[7] Dupuis J. Endothelin: setting the scene in PAH. Eur Respir. Rev. 2007; 16: 3-7.

[8] Galie N, Manes A, Branzi A. The endothelin system in pulmonary arterial hypertension. Cardiovasc. Res., 2004; 61: 227-37.

[9] Tuder RM, Cool CD, Geraci MW, et al. Prostacyclin synthase expression is decreased in lungs from patients with severe pulmonary hypertension. Am. J. Respir. Crit. Care Med. 1999; 159: 1925-32.

[10]Atz AM, Adatia I, Wessel DL. Rebound pulmonary hypertension after inhalation of nitric oxide. Ann. Thorac. Surg., 1996; 62: 1759-64.

[11]Montani D, Gunther S, Dorfmuller P, et al. Pulmonary arterial hypertension. Orphanet J Rare Dis., 2013; 8: 97. 
[12]Olivier S, Nicholas WM. Pathways in pulmonary arterial hypertension: the future is here. Eur. Respir. Rev., 2012; 21: 321-27.

[13]Taichman DB, Ornelas J, Chung L, et al. Pharmacologic therapy for pulmonary arterial hypertension in adults: CHEST guideline and expert panel report. Chest, 2014; 146: 449-75.

[14]Sun W, Chan SY. Pulmonary Arterial Stiffness: An Early and Pervasive Driver of Pulmonary Arterial Hypertension. Front. Med., 2018; 5: 204.
[15]Lan NSH, Massam BD, Kulkarni SS, Lang CC. Pulmonary Arterial Hypertension: Pathophysiology and Treatment. Diseases, 2018; 6(2); 38. doi: 10.3390/diseases6020038.

[16]Huertas A, Tu L, Guignabert C. New targets for pulmonary arterial hypertension: going beyond the currently targeted three pathways. Curr. Opin. Pulm. Med., 2017; 23(5): 377-85. doi: 10.1097/MCP.0000000000000404. 